\title{
Numerical analysis with experimental verification to predict outdoor power conversion efficiency of inverted organic solar devices
}

\begin{abstract}
Inverted organic solar cell (IOSC) devices with different volume ratios of $\operatorname{In}_{2} \mathrm{~S}_{3}$ nanoparticles have been studied under local spectral irradiances in Malaysia with respect to that of AM1.5G. The J-V curves of encapsulated IOSC devices were measured outdoor using an Ivium Potentiostat and local spectral irradiances were acquired using an AVANTES spectrometer concurrently. All of the IOSC devices experienced significant improvement in power conversion efficiency (PCE) under the both local sunny and cloudy conditions with respect to the AM $1.5 \mathrm{G}$, by $22-35 \%$ and $31-65 \%$, respectively. From spectral analysis, the area under the graph of spectral irradiance in UV-visible region is significantly higher compared to infrared region for both local sunny and cloudy conditions, by $44.6 \%$ and $55.9 \%$, respectively, while it is only recorded as $12.9 \%$ for AM 1.5G. Last but not the least, we have successfully verified the numerical analysis to predict device performance by comparing the simulated and measured PCE values for different irradiance intensities whereby the prediction of PCE is better under sunny condition with a deviation of 3.4-10.8\% compared to cloudy conditions, with deviation of $28.9-30.5 \%$.
\end{abstract}

Keyword: Inverted organic solar cell; Local spectral irradiance; J-V characteristic curve; Power conversion efficiency; Standard AM 1.5 G spectrum; P3HT:PC 61 BM 